\title{
Magnum Opus: Clustered Regularly Interspaced Short Palindromic Repeats Biology and Prokaryotic Gene Silencing
}

\author{
${ }^{1}$ Prem Saran Tirumalai and ${ }^{2}$ Ashim Kumar Bagchi \\ ${ }^{1}$ Department of Biotechnology, \\ Uttam Institute of Technology and Management, Runakta, Agra-282005, India \\ ${ }^{2}$ Department of Physiology, Faculty of Medicine, University of Manitoba, Winnpeg, Canada
}

Received 2013-09-24, Revised 2013-09-25; Accepted 2013-10-05

\begin{abstract}
Gene Silencing was a technology that was established in eukaryotic system a decade ago and is being used as a research tool widely. However, prokaryotic gene silencing was not workable, till recently a team of researchers from the University of Georgia have proved it possible. Where they have shown that short motif sequences determines the targets of the prokaryotic Clustered Regularly Interspaced Short Palindromic Repeats (CRISPR) defence system is regulated by RNA guided Cas protein complex. Thus role of CRISPR system in microbial defense against foreign genetic material (Plasmid or Phages) is an important milestone in the field of microbial molecular biology/biotechnology. These findings will make it easier to understand the significance of a gene, metabolically or physiologically. The revelation by this novel finding by core group of researcher is indeed, Mangum opus. This article is a commentary, to bring to light, prokaryotic gene silencing as one of the latest advances in prokaryotic science.
\end{abstract}

Keywords: Gene Silencing, Prokaryotes, CRISPR, RNAi, Microbial Immunity

\section{INTRODUCTION}

For all of us, working to unfold the puzzling and intriguing world of genes in prokaryotes, their sequential makeup and their significance, have reasons to breathe easy and stretch, now. Gene Silencing a promising technology that fetched Nobel Prize to the discoverers Craig Mello and Andrew Fire, that was confined to eukaryotic system, ever since its discovery in 1998, is now feasible in prokaryotic systems (Hale et al., 2012). Hale et al. (2012) supported by state that, rationally designed RNAs can work with bacterial immunity to silence target genes in prokaryotes (Rusk, 2012). It's time to crack the code!

Understanding the physiological or metabolic role of a gene was/is crucial to many of biological processes. Till the recent revelations on prokaryotic gene silencing, the role of a gene in a prokaryotic system could be determined either by 'knout-out' or by 'mutational studies'. RNA interference (RNAi/Post-transcriptional Gene Silencing) could not be applied to prokaryotes.

\subsection{Gene Silencing Basics}

Post-transcriptional gene modification and its silencing is mediated by short interfering RNA (siRNA). This is believed to be a naturally occurring biological process, which is conserved in plants and animals (Keates et al., 2008). The innate nature of RNAi owing to posttranscription rather than transcription level which has been adopted from dsRNA through the suppression of epigenetic or against intronic regions corresponding to transcribed (Fire et al., 1998; Hammond et al., 2001). RNA interference (RNAi) in mammalian cells otherwise termed as 'Gene Silencing' has been stated to have evolved as an immune response to viral invasion (Caplen et al., 2001; Hammond et al., 2001). This understanding led to the discovery of artificially synthesized siRNA and Runakta, Agra-282005, India 
synthetic siRNA as we know, that revolutionized our understanding to the functions of genes.

Gene silencing that have described in prokaryotes is different from the gene silencing that happens in eukaryotic system, with reference to exogenous siRNA (Hale et al., 2008; 2009; 2012). In the eukaryotes, when siRNA molecules are applied exogenously, the siRNA duplexes are unwound by RNA-Induced Silencing Complex (RISC). In this process, the sense strand, homologous to the target gene transcript (mRNA) is eliminated. The remaining anti-sense strand that is complementary to the target gene transcript, along with the RISC complex, binds to the transcript. The RISC complex binds to the complementary region of the target mRNA through the antisense siRNA strand. RISC complex then cleaves the target mRNA molecule using Argonaute-2 protein activity (Hale et al., 2008; Wiedenheft et al., 2011a; 2011b; 2012).

\subsection{The 'Crispr' Purpose in Prokaryotes}

Microbes have a unique defensive mechanism through CRISPR system, which acts as a determinant of genetic interference pathway against phages and plasmids (Labrie et al., 2010; Garneau et al., 2010; Sapranauskas et al., 2011). Since viruses show diverse antigenic variations (Rohwer, 2003; Pignatelli et al., 2008; Hambly and Suttle, 2005) caused due to rapid mutation, they easily evade the host immune system. Immunity to these viral DNA is a challenging issue and hence CRISPR-cas system participating in disintegration and immobilization of their gene activity, a subject of interest. Through specific nuclease activity of CRISPR associated Cas the foreign DNA/plasmid is spliced into smaller fragments. These fragments are then transcribed. The transcribed sequences are further utilized as noncoding RNA template for interference, invasion and disintegration (Hale et al., 2009). Thus immunity in microbes is a RNA-encoded memory response, which is always acquired and adaptive (Barrangou et al., 2007; Deveau et al., 2008; Koonin and Makarova, 2009; Horvath and Barrangou, 2010; Marraffini and Sontheimer, 2010; Bhaya et al., 2011; Shah and Garrett, 2011). Unlike, in eukaryotes, innate response is not at all necessary in prokaryotes to achieve protection from invasion of such foreign particles. Because of their unique CRISPR system, they do not require prior contact of these foreign particles in order to react against them. CRISPR-Cas system a new-generation biological tool for the development of gene-based targeted therapy is an immune mechanism adopted by microbes against invading nucleic acid either in the form of plasmids or encapsulated in a virus.

\subsection{The 'Crispr-Cas' Mechanism}

CRISPR-Cas-mediated defense mechanism has been widely discussed in terms of immunization by controlling invasion and integration of foreign particles and immunity (Horvath and Barrangou, 2010; Babu et al., 2011) through the development of inherited memory (Oost et al., 2009). However, in the larger interest it is worthwhile discussing the stage-wise mechanism of the system. Previous data reveals that CRISPR-Cas mediates both active and passive immunity through different immunization process (Fig. 1). The process involves (i) recognition of invasive particles, (ii) propagation of repeat sequence selection of spacer sequence and integration into CRISPR loci, (iii) transcription, includes editing of spacer sequence and synthesis of multiple copies of noncoding RNA sequence with the help of multifunctional nucleases, Cas1, Cas2, Cas3, finally, (iv) Cas specifically cleaves and splice these invasive foreign phage DNA or plasmid and acquire immunity against invasive genetic materials is thus developed (Wiedenheft et al., 2009; 2011a). In terms of active immunity host directly comes in contact with these foreign particles and integrates while passive immunity develops through transfer of predisposed acquired information from generation after generation.

CRISPR are regions in a prokaryotic genome that contains multiple short direct repeats separated by spacer genes (Hale et al., 2009; 2012). CRISPR confers immunity in prokaryotes against invading exogenous genetic materials such as plasmid or phage (Mojica et al., 2009). When exogenous genetic material invades the prokaryotic cell, they are processed by a group of genes that are associated with the CRISPR region. These genes are termed as the cas genes. The Cas proteins derived from the cas genes, break down the invading genetic material into small fragments of 25 to 30 nucleotide base pairs. These fragments are then inserted into the CRISPR region. RNA molecules (along with the exogenous inserts) derived of constitutive transcription of the CRISPR are further processed by Cas proteins to yield small RNA fragments composed of the exogenous genetic material flanked with a repeat sequences. These RNA fragments are complementary to the invading genetic material. These RNA fragments forms an effector complex with Cas proteins (similar to the RISC complex in eukaryotes). This effector complex, due to the complementary, binds and silences the invading exogenous genetic material (Hale et al., 2012; Caplen et al., 2001; Wiedenheft et al., 2011b; 2012). 
Prem Saran Tirumalai and Ashim Kumar Bagchi / American Journal of Immunology 9 (4): 110-115, 2013

Step 1: Recognition, Repeat Sequence Synthesis and Editing

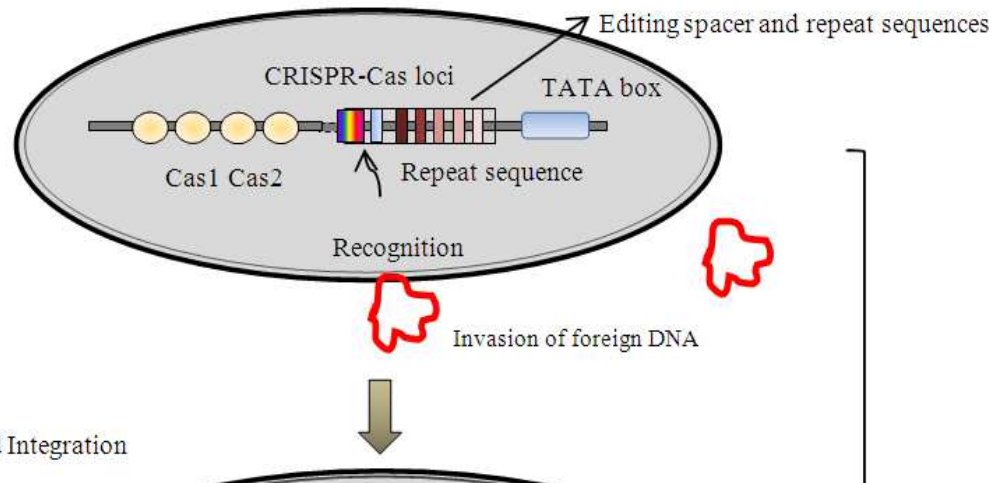

Step 2: Spacer re-arrangement and Integration

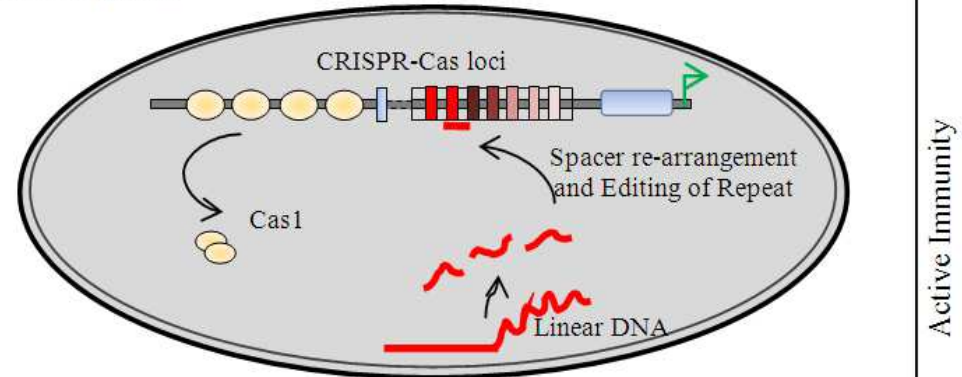

Step 3: Transcription, Interloping and Degradation

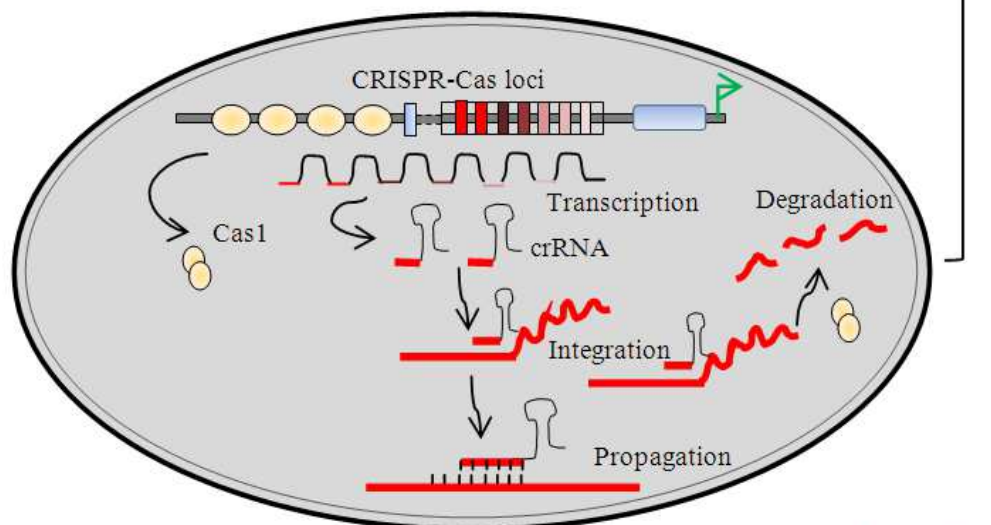

Step 4: Immunity and Memory
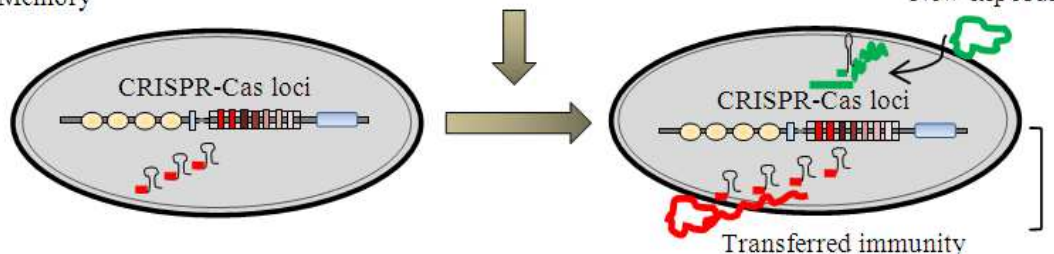

Passive Immunity

Fig. 1. CRISPR-Mediated active and passive immunity may achieve in four different steps: Step 1 includes recognition of invasive foreign DNA and synthesis of repeat sequences, step 2 includes re-arrangement of spacer and editing in repeat sequences with the help of multifunctional protein Cas1. Cas1 splices into small pieces are now transcribed into RNA encoded to these sequences and make copies of non-coded RNA that integrates with foreign DNA. Step3: These non-coding RNA utilizes to interfere in foreign DNA invasion and its degradation. Step 4: In this process microbes develops memory for specific DNA exposed to it in order to acquire immunity and transfer this immunity passively from one generation to another 


\subsection{The Discovery}

The idea about the Clusters of Regularly Palindromic Repeat (CRISPR) locus developed in (Mojica et al., 1995) from the discovery of short sequence in between the 28-30 nucleotide (nt) long repeats in Escherichia coli K-12 chromosome (Ishino et al., 1987; Nakata et al., 1989). Since then, there have been several reports on cloning and sequencing of these genes in prokaryotes (Hermans et al., 1991; Kawarabayasi, 1998; Kawarabayasi et al., 1999; Hoe, 1999; Mojica et al., 2000). However, its science and significance towards application have been established only after the year 2000 (Bolotin et al., 2005; Mojica et al., 2000; 2005; Pourcel et al., 2005; Makarova et al., 2006).

\subsection{Codicil}

Ultimate goal of all medical research is to find out the target molecule as a key regulator of immune system by means of either activating host immune response or by inhibiting microbial response. This recent discovery about CRISPR system in bacteria and archaea brought revolution in the field of genetic engineering and biotechnology. Molecular mechanisms of these microbial effects are widely regulated by nucleic acid followed by alteration of hyper-variable gene patterns where noncoding RNA from CRISPR loci provides acquired immunity against non-specific sequence (Karginov and Hannon, 2010). Putative regulatory role of these noncoding RNA in CRISPR system provides important information about naturally controlled defensive mechanism, which may help in developing gene-specific model in medical research. Using this crisp CRISPR system, we can insert any target gene of interest that encoded for immunity by such regulated CRISPR may be achieved through siRNA or miRNA in eukaryotic animals or mammalian (Makarova et al., 2006).

This technique of Gene silencing has proved to be a new-generation high throughput biological tool in understanding the transcriptional activity of janus-kinase (Jak)-Signal Transducer and Activator of Transcription (STAT) or Nuclear Factor-kappaB (NFkB) and also functional mechanism of other signalling pathway (Sharma and Rao, 2009).

\section{CONCLUSION}

Observation by Hale and his colleagues using CmrcrRNA complexes, has given a workable solution to targeted destruction of gene transcripts (posttranscriptional) in prokaryotes.
This finding is an important milestone in the field of microbial molecular biology/biotechnology, as it will make it easier to understand the significance of a gene, metabolically or physiologically. The revelation by this novel finding by core researcher is indeed, Mangum opus. It is high time researchers in the domain, young and old alike, embraced this technology for deciphering the significance of genes and the information contained in them.

\section{REFERENCES}

Babu, M., N. Beloglazova, R. Flick, C. Graham and T. Skarina et al., 2011. A dual function of the CRISPRCas system in bacterial antivirus immunity and DNA repair. Mol. Microbiol., 79: 484-502. DOI: 10.1111/j.1365-2958.2010.07465.x

Barrangou, R., C. Fremaux, H. Deveau, M. Richards and P. Boyaval et al., 2007. CRISPR provides acquired resistance against viruses in prokaryotes. Science, 315: 1709-1712. DOI: 10.1126/science. 1138140

Bhaya, D., M. Davison and R. Barrangou, 2011. CRISPR-Cas systems in bacteria and archaea: Versatile small RNAs for adaptive defense and regulation. Annu. Rev. Genet., 45: 273-297. DOI: 10.1146/annurev-genet-110410-132430

Bolotin, A., B. Ouinquis, A. Sorokin and S.D. Ehrlich, 2005. Clustered Regularly Interspaced Short PAlindrome Repeats (CRISPRs) have spacers of extrachromosomal origin. Microbiology, 151: 25512561. DOI: $10.1099 /$ mic.0.28048-0

Caplen, N.J., S. Parrish, F. Imani, A. Fire and R.A. Morgen, 2001. Specific inhibition of gene expression by small double-stranded RNAs in invertebrate and vertebrate systems. PNAS, 98: 9742-9747. DOI: 10.1073/pnas. 171251798

Deveau, H., R. Barrangou, J.E. Garneau, J. Labonte and P. Boyaval et al., 2008. Phage response to CRISPRencoded resistance in Streptococcus thermophilus. J. Bacteriol., 190: 1390-1390. DOI: 10.3410/f.1098565.554664

Fire, A., S. Xu, M.K. Montgomery, S.A. Kostas and S.E. Driver et al., 1998. Potent and specific genetic interference by double-stranded RNA in Caenorhabditis elegans. Nature, 391: 806-811. DOI: $10.1038 / 35888$

Garneau, J.E., M.E. Dupuis, M. Villion, D.A. Romero and R. Barrangou et al., 2010. The CRISPR/Cas bacterial immune system cleaves bacteriophage and plasmid DNA. Nature, 468: 67-71. DOI: 10.1038/nature09523 
Hale, C., K. Kleppe, R.M. Terns and M.P. Terns, 2008. Prokaryotic silencing (psi)RNAs in Pyrococcus furiosus. RNA, 14: 2572-2579. DOI: 10.1261/rna.1246808

Hale, C.R., P. Zhao, S. Olson, M.O. Duff and B.R. Graveley et al., 2009. RNA-guided RNA cleavage by a CRISPR RNA-Cas protein complex. Cell, 139: 945-956. DOI: 10.1016/j.cell.2009.07.040

Hale, C.R., S. Majumdar, J. Elmore, N. Pfister, M. Compton et al., 2012. Essential features and rational design of CRISPR RNAs that function with the Cas RAMP module complex to cleave RNAs. Mol. Cell, 45: 292-302. DOI: 10.1016/j.molcel.2011.10.023

Hambly, E. and C.A. Suttle, 2005. The viriosphere, diversity and genetic exchange within phage communities. Curr. Opin. Microbiol., 8: 444-450. DOI: 10.1016/j.mib.2005.06.005

Hammond, S.M., A.A. Caudy and G.J. Hannon, 2001. Post-transcriptional gene silencing by doublestranded RNA. Nat. Rev. Genet., 2: 110-119. PMID: 11253050

Hermans, P.W., D. Van Soolingen, E.M. Bik, P.E. De Haas and J.W. Dale et al., 1991. Insertion element IS987 from Mycobacterium bovis BCG is located in a hot-spot integration region for insertion elements in Mycobacterium tuberculosis complex strains. Infect. Immun., 59: 2695-2705. PMID: 1649798

Hoe, N., 1999. Rapid molecular genetic subtyping of serotype M1 group A Streptococcus strains. Emerg. Infect. Dis., 5: 254-263. PMID: 10221878

Horvath, P. and R. Barrangou, 2010. CRISPR/Cas, the immune system of bacteria and archaea. Science, 327: 167-170. DOI: 10.1126/science. 1179555

Ishino, Y., H. Shinagawa, K. Makino, M. Amemura and A. Nakata, 1987. Nucleotide sequence of the iap gene, responsible for alkaline phosphatase isozyme conversion in Escherichia coli and identification of the gene product. J. Bacteriol., 169: 5429-5433.

Karginov, F.V. and G.J. Hannon, 2010. The CRISPR system: Small RNA-guided defense in bacteria and archaea. Mol. Cell, 37: 7-19. DOI: 10.1016/j.molcel.2009.12.033

Kawarabayasi, Y., 1998. Complete sequence and gene oganization of the genome of a hyperthermophilic archaebacterium, Pyrococcus Horikoshii OT3. DNA Res., 5: 55-76. PMID: 9679194

Kawarabayasi, Y., Y. Hino and H. Horikawa, 1999. Complete genome sequence of an aerobic hyperthermophilic crenarchaeon, Aeropyrum pernix K1. DNA Res., 6: 83-101. PMID: 10382966
Keates, A.C., J. Fruehauf, S. Xiang and C.J. Li, 2008. Trans kingdom RNA interference: A bacterial approach to challenges in RNAi therapy and delivery. Biotechnol. Gene. Eng. Rev., 25: 113-128. PMID: 21412352

Koonin, E.V. and K.S. Makarova, 2009. CRISPR-Cas: An adaptive immunity system in prokaryotes. F1000 Biol. Rep., 1: 95-95. PMID: 20556198

Labrie, S.J., J.E. Samson and S. Moineau, 2010. Bacteriophage resistance mechanisms. Nat. Rev. Microbiol., 8: 317-327. DOI: 10.1038/nrmicro2315

Makarova, K.S., N.V. Grishin, S.A. Shabalina, Y.I. Wolf and E.V.A. Koonin, 2006. Putative RNAinterference-based immune system in prokaryotes: Computational analysis of the predicted enzymatic machinery, functional analogies with eukaryotic RNAi and hypothetical mechanisms of action. Biol. Direct., 1: 7-7. DOI: 10.1186/1745-6150-1-7

Marraffini, L.A. and E.J. Sontheimer, 2010. CRISPR interference: RNA-directed adaptive immunity in bacteria and archaea. Nat. Rev. Genet., 11: 181-190. DOI: $10.1038 / \mathrm{nrg} 2749$

Mojica, F.J., C. Diez-Villasenor, E. Soria and G. Juez, 2000. Biological significance of a family of regularly spaced repeats in the genomes of Archaea, Bacteria mitochondria. Mol. Microbiol., 36: 244246. DOI: 10.1046/j.1365-2958.2000.01838.x

Mojica, F.J., C. Diez-Villasenor, J. Garcia-Martinez and C. Almendros, 2009. Short motif sequences determines the targets of the prokaryotic CRISPR defence system. Microbiology, 155: 733-740. DOI: 10.1099/mic.0.023960-0

Mojica, F.J., C. Diez-Villasenor, J. Garcia-Martinez and E. Soria, 2005. Intervening sequences of regularly spaced prokaryotic repeats derive from foreign genetic elements. J. Mol. Evol., 60: 174-182. DOI: 10.1007/s00239-004-0046-3

Mojica, F.J., C. Ferrer, G. Juez and F. Rodriguez-Valera, 1995. Long stretches of short tandem repeats are present in the largest replicons of the Archaea Haloferax mediterranei and Haloferax volcanii and could be involved in replicon partitioning. Mol. Microbiol., 17: 85-93. DOI: 10.1111/j.13652958.1995.mmi 17010085.x

Nakata, A., M. Amemura and K. Makino, 1989. Unusual nucleotide arrangement with repeated sequences in the Escherichia coli K-12 chromosome. J. Bacteriol., 171: 3553-3556. PMID: 2656660 
Oost, J.V.D., M.M. Jore, E.R. Westra, M. Lundgren and S.J. Brouns, 2009. CRISPR-based adaptive and heritable immunity in prokaryotes. Trends Biochem. Sci., 34: 401-407. PMID: 19646880

Pignatelli, M., G. Aparicio, I. Blanquer, V. Hernandez and A. Moya et al., 2008. Metagenomics reveals our incomplete knowledge of global diversity. Bioinformatics, 24: 2124-2125. DOI: 10.1093/bioinformatics/btn355

Pourcel, C., G. Salvignol and G. Vergnaud, 2005. CRISPR elements in Yersinia pestis acquire new repeats by preferential uptake of bacteriophage DNA and provide additional tools for evolutionary studies. Microbiology, 151: 653-663. DOI: 10.1099/mic.0.27437-0

Rohwer, F., 2003. Global phage diversity. Cell, 113: 141-141. PMID: 12705861

Rusk, N., 2012. Prokaryotic RNAi. Nat. Methods, 9: 220-221. DOI: 10.1038/nmeth.1916

Sapranauskas, R., G. Gasiunas, C. Fremaux, R. Barrangou and P. Horvath et al., 2011. The Streptococcus thermophilus CRISPR/Cas system provides plasmid immunity in Escherichia coli. Nucl. Acids Res., DOI: 10.1093/nar/gkr606

Shah, S.A. and R.A. Garrett, 2011. CRISPR/Cas and $\mathrm{Cmr}$ modules,mobility and evolution of adaptive immune systems. Res. Microbiol., 162: 27-38. DOI: 10.1016/j.resmic.2010.09.001
Sharma, S. and A. Rao, 2009. RNAi screening: Tips and techniques. Nature Immunol., 10: 799-804. DOI: 10.1038/ni0809-799.

Wiedenheft, B., E.V. Duijn, J.B. Bultema and S.P. Waghmare, 2011a. RNA-guided complex from a bacterial immune system enhances target recognition through seed sequence interactions. PNAS, 108: 10092-10097. DOI: 10.1073/pnas.1102716108

Wiedenheft, B., G.C. Lander and K. Zhou, $2011 \mathrm{~b}$. Structures of the RNA-guided surveillance complex from a bacterial immune system. Nature, 477: 486489. DOI: $10.1038 /$ nature 10402

Wiedenheft, B., K. Zhou, M. Jinek, S.M. Coyle and W. Ma et al., 2009. Structural basis for DNase activity of a conserved protein implicated in CRISPRmediated genome defense. Structure, 17: 904-912. DOI: $10.1016 /$ j.str.2009.03.019

Wiedenheft, B., S.H. Sternberg and J.A. Doudna, 2012. RNA-guided genetic silencing systems in bacteria and archaea. Nature, 482: 331-338. DOI: 10.1016/j.str.2009.03.019 